\title{
Supplement to the knowledge of the systematics of Morus in Japan
}

\section{By Teikichi HotTA*}

\section{堀田頑吉： 邦產野生桑属の分類学的知見補遺}

Key to the species, varieties and forms of Morus found in wild.

1. Style long, stigma divided into two parts at its apex......Sect. I. Dolichostylae KoIDz.

Style none or very short with sessile or subsessile stigma divided into two parts

Sect. II. Macromorus KoIDz. (Morus Miyabeana HotTA).

2. Apex of the leaf caudate rarely acuminate... Morus australis POIRET.

Apex of the leaf acuminate or acute rearly caudate or subulate.

3. Upper surface of the leaf is conspicuously lustrous, and has a dark green colour.

Upper surface of the leaf is usually dull green rarely slightly lustrous. var. glabra KoIDz.

. Apex of the leaf subulate, caudate or longiacuminate. var. typica HotTA

Apex of the leaf acuminate rarely subtricuspidate. 5

5. Upper surface of the leaf rather scabrous or smooth. 6 Upper surface of the leaf asperate. .var. asperata HoTTA

6. Under surface with a few short hairs. 8

Under surface with conspicuously hairs.

7. Margin of the leaf arguto-serrulate or dentato-serrulate. var, diabolica HotTA

Margin of the leaf dentato-serrato or largely dentato-serrato.

8. Leaf variously lobate, apex of the leaf acuminate and subtricuspidate......var. vestita KoIDz. Leaf usually not lobate or 2-4 lobate, apex of the leaf acuminate or acute. var. pubescens (YENDO) HOTTA

9. Apex of the leaf subulate. .var. subulata HoTTA Apex of the leaf caudate. var. caudatifolia KoIDZ

10. Leaf lobate or not lobate. In former case usually the length of the lobe is about half-way between the margin and midlib. f. normalis HoTTA

Lobe are usually rather deep, and the segments are very anormal.......... anormalis HoTTA

Sect. I. Dolichostylae Koidzumi Imp. Sericult. Exp. Stat., 2, No. 1, 3 (1923).

1). Morus bombycis KoIdzumi Bot, Mag. Tokyo, 24, 313 (1915).

Nom. Jap. Yamaguwa.

Deep acknowledement is due to prof. Emer. K. MIYABE and S. ITo who have given constant guidance and to Dr. M. TATEWAKI who have rendered kind advice and to T. SAKAMOTo and T. IsAGo who helped the writer in many way.

* Kyoto Univertity of Industrial Arts and Textile Fibers, Japan. 
Distr. Saghalien, Yezo, Honsiu, Sikoku, Kiusiu and Korea.

var. Hamadai Hotт, var. nov.

Folia varie incisa vel caceria, subtus tomentosa, apice caudata, subtricuspidata, magno dentato-serrata.

Nom. Jap. Kekameba-guwa (nov.).

Distr. Honsiu.-Hab. Prov. Rikutiu : Kayakariba, Iwasaki, Waka (G. Kobayashi, no. 8791).

Remarks. Leaf is variously incised or lacerate, under surface tomentose, apex caudate and subtricuspidate, margin largely dentato-serrate.

var. asperata Hoтt A, Bot Mag. Tokyo, 52, No. 614, 79 (1938).

Folia supra valde asperata rare scaberrima, apice caudata vel longiacuminata.

Nom. Jap. Zaraba-guwa.

Distr. Honsiu, Sikoku, Kiusiu.-Hab. Prov. Rikutiu, Nonogami, Kondaiti, Ninohe (T. IsAGo, no. 48990); Hirasimizu, Simohei (T. HotTA, no. 41999).

var. diabolica HоттA, Bot. Mag. Tokyo, 51, 691 (1937).

Folia irregulariter duplo-lobata, margine arguti-serrulata vel dentato-serrulata, apice leviter incurva.

Nom. Jap. Oniyama.guwa.

Distr. Honsiu.-Hab. Prov. Rikutiu: Kayakariba, Iwasaki, Waka, (T. IsaGo, no. 49001), Akasaki, Kesennuma (T. IsAGo, no. 49002).

var. vestita KoInz., Bull. Imp. Sericult. Exp. Stat., 2, No. 1, 13 (1923).

Folia varie lobata, subtus tomentosa. Apice plerumque lobi fere subito cuspidatus. Petiolus fere tenuis.

Nom. Jap. Kirebakeyama-guwa.

Distr. Honsiu.-Hab. Prov. Rikutiu: Kannonbayasi, Hareyama, Kunohe (T. IsAgo, no. 48994).

var. pubescens Hotta, Trans. Sapporo Nat. Hist. Soc., 14, No. 3, 201, t. 6, c (1936). (new to Rikutiu).

Ramuli plerumque tenues, fere declinati. Folia subtus insigniter pubescentia.

Nom. Jap. Keyama-guwa.

Distr. Yezo and Honsiu-Hab. Prov. Rikutiu: Iwasaki, (T. Hotta, no. 8001);

Takekoma, Kesen (T. IsAGo, no. 48002).

var. subulata Hотт,, Bot. Mag. Tokyo, 51, 692 (1937).

Folia plerumque 2-4 lobata vel non lobata, apice subulata, margine dentatoserrata, basi cuneata vel truncata rare aperte cordata, petiolo $2.5-4 \mathrm{~cm}$. longo.

Nom. Jap. Kirisakiyama-guwa.

Distr. Honsyu.--Hab. Rikutiu : Terata, Waki (T. HotтA, no. 8291).

var. caudatifolia KoIDzumI, Kuwazoku Shokubutsuko, 33 in nota sub. $M$. bombycis, (1919). 
Folia; apice caudata subito elongata.

Nom. Jap. Onaga-guwa.

Distr. Saghalien, Yezo, Honsiu, Sikoku, Kiusiu and Korea.-Hab. Prov. Rikutiu: Yonesato, Yeri (T. IsAGo, no. 9974) (New to Rikutiu).

f. anormalis Hотт,, Trans. Sapporo. Nat. Hist. Soc., 14, 203 (1936).

Segmenta folii valde anormalia; petiolo plerumque omnino fere insigniter pubescentia.

Nom. Jap. Okinayama-guwa.

Distr. Yezo and Honsiu.-Hab. Prov. Rikuzen: Akazaki, Kesen (T. IsAGo, no. 48997).

2). Morus australis Poiret, Lamarck, Ency. Method. Bot., 4, 380 (1797).

Nom. Jap. Suima-guwa.

Distr. Formosa, Riukiu, Kiusiu (Ôsumi, Tanegasima, Yakusima, Tokunosima).

Hab. Prov. Ốsumi : Ôsima, Nago (T. Hotta, no. 34474).

Remarks. The leaf of the one (A) is less lustrous then of the other (B); the lateral vein of $A$ is alternate and that of $B$ is oppsite; the margin of $A$ is dentatoserrate and that of $B$ is crenato-dentate; the base of $A$ is cordate and that of $B$ is truncate. There are two kinds of $\mathrm{A}$, wile the one falls late Autumn and the other is evergreen, $B$ is desiduous.

var. glabra KoIDzumi, Bull. Imp.

Sericult. Exp. Stat. 2, No. 1, (1932)

Morus cuspidata W ALL. var. Loochooensis YENDO, Traite sur la cult. du Mūr. au Jap., 30 (1930). nom. seminud.

Folia glaberrima nitida valde viridia.

Nom. Jap. Terihasima-guwa (T.

Hotta, no. 1704).

Distr. Kiusiu, Riukiu and Formosa.

Hab. Hiyuga: Ôsima, Minaminaka. (New to Kiusiu).

Sect. II Macromorus KoIDzUMI, 3, (1923).
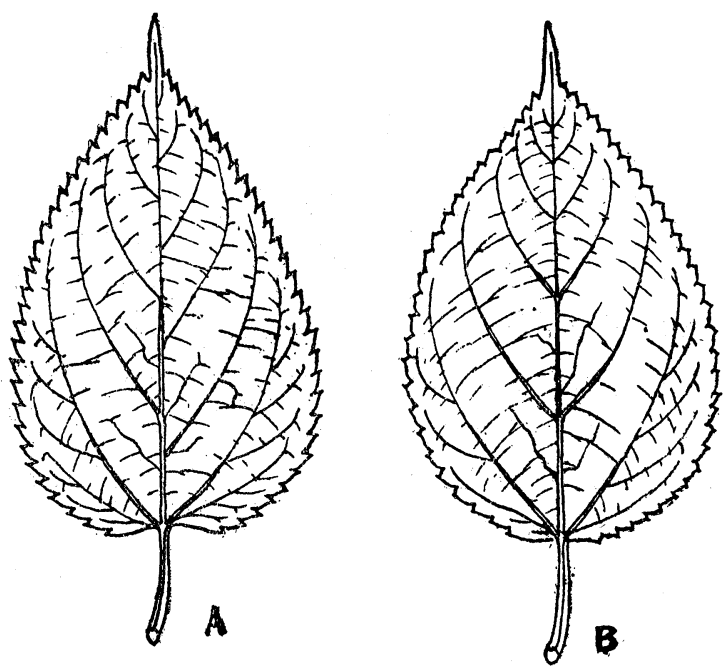

Fig. 1. Two types in leaf of Morus australis POIRET

\section{3.) Morus Miyabeana HotтA, sp. nov.}

Arbor; cortice fusca vel fuliginosa, lenticellis lineari-elliptica vel rotundata, cinereofusca, dispersia. Ramuli fere declinata. Gemmae ovales, rufae. Folia ovatolanceolata vel ovata rarissime crassa, adulta supra valde viridia non nitida, fere 
glabra rare fere pilosa, apice acuminata vel acuta, margine dentato-serrata vel

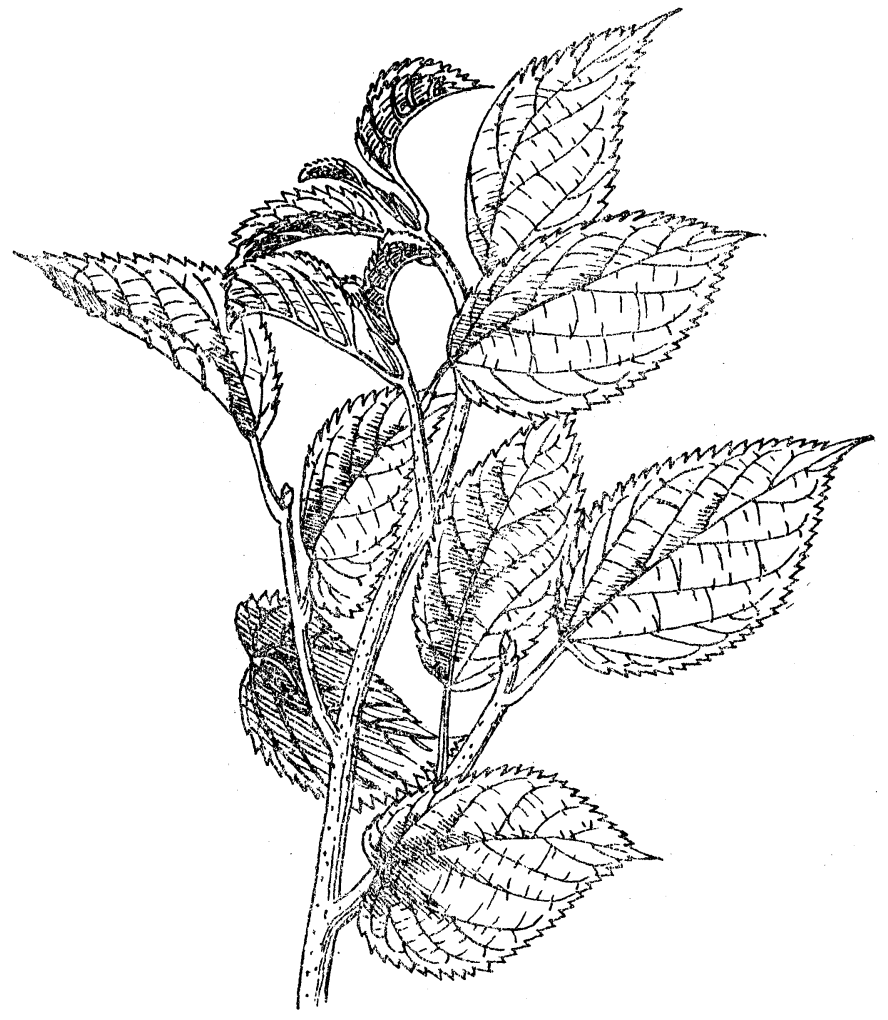

Fig. 2. Morus Miyabeana HoTTA dentato-serrulata, basi truncata vel rotundata. Lamina fere $8 \mathrm{~cm}$. longa, 5 cm. lata, nervis axillaribus 4-5; nervis majoribus ex petiolo divisis, nervis capillaribus infra nervos axillares promissis petiolus tenuis circ. $3 \mathrm{~cm}$. longus, sulco valde angustus. Stipulae circ. $1 \mathrm{~cm}$. longae linearilaceolatae caducae. Cystolithus apice fere acuto. Tepala perianthii masculi 4, apice obtusa extus supra ciliolata, 2 mm. longa. Amenta mascula fere pendula, cylindrica, $1-1.5 \mathrm{~cm}$. longa, pedicellata pilosa filiformia brev. issima. Tepala perianthii feminei ovario approximata. Ovarium circ. 0.7

mm. longum. Stylus breirs $0.2 \mathrm{~mm}$. stigmatibus 2 eum sessilibus, intus densissime pilosis. Spica fructifera circ. 3-5 $\mathrm{mm}$. longa, primo rubra mox atrata.

Nom. Jap. Amakusa-guwa (nov.).-Nom. Vern. Yama-guwa.

Distr; Kiusiu.-Hab. Higo: Honto, Amakusa (T. HotтA, no. 7, typus).

Remarks. This species is closely akin to Morus macroura MiQ. in the length of its style and in the leaf form, but the former is less tomentose than that of the latter.

\section{摘 要}

Morus Miyabeana の 1 新種和よび Morus bombycis KoIDz. var. Hamadai の 1 新变種を創定した。 ま Morus australis POIRET var. glabra KOIDz. を九州冰和いて発見し Morus bombycis KoIDz. K fo $\checkmark \tau$ var. diabolica HotTA, var. pubescens HotTA, var. asperata HоTтA, var. subulata HоттA. var. vestita KoIDz. の 5 変種が陸中に産し, f. anormalis HOTTA の 1 品種が陸前に産するととを確認し $k_{0}$ 